Pacific Journal of Mathematics

ON A CLASS OF CONTRACTIVE PERTURBATIONS OF 


\title{
ON A CLASS OF CONTRACTIVE PERTURBATIONS OF RESTRICTED SHIFTS
}

\author{
Joseph A. BALL AND ARthur LUbin
}

The Sz.-Nagy-Foias model theory uses generalized restricted shifts as canonical models for contractions in Hilbert space. This paper considers a class of contractive and unitary perturbations of a generalized restricted shift acting on a Sz.-Nagy-Foias space generated by an analytic operator-valued function $S(z)$ whose values are contractions on a separable Hilbert space. The spectra and characteristic functions of the perturbations are computed and related to the original operator. When the perturbation is unitary, a unitary equivalence to multiplication by $e^{i \theta}$ on $L^{2}(\mu)$, for an operatorvalued measure $\mu$, is given.

In [2], D. N. Clark studied the one-dimensional unitary perturbations of restricted shifts in $H^{2}$, i.e. $S(z)$ a scalar inner function, and in [3], he announced results for the case where $S(z)$ is an arbitrary scalar (characteristic) function. The general unitary perturbations are implicit in work of de Branges and Rovnyak [1], though in the context of the de Branges-Rovnyak model theory rather than the Sz.-Nagy-Foias. P. A. Fuhrmann [5] considered a class of completely nonunitary and unitary perturbations for the case of $S(z)$ an inner function on a finite-dimensional space. In this case, the maps considered are always compact perturbations. Here we generalize results of [5] and [2]. We will follow the general outline of [5], and we correct a minor error occurring there so our description of the perturbations in the general case is actually as sharp as in the finite-dimensional case. As was pointed out in [5], these perturbations have applications to the theory of stability of linear control systems.

1. Preliminary results. For notation, let $C$ and $C_{*}$ be separable Hilbert spaces, and let $L^{2}(C), L^{2}\left(C_{*}\right), H^{2}(C), H^{2}\left(C_{*}\right)$ denote the standard vector-valued Lebesgue and Hardy spaces defined on the unit circle. (See [6] or [8] for general references.) We will use " $t$ " to denote the argument of a function (vector or operator valued) defined on the unit circle, and for analytic functions, we will freely identify $h(t)$ on the circle with its extension to the disc, denoted $h(z) . \quad S$ will denote a fixed purely contractive analytic operator-valued function from $C$ to $C_{*}$, i.e. $S(z): C \rightarrow C_{*},\|S(z)\| \leqq 1$ for all $|z|<1$ and $\|S(0) c\|<$ $\|c\|$ for all $c \in C$, and let $\Delta(t)=\left(I-S(t)^{*} S(t)\right)^{1 / 2}$. (Note that this denotes the unique positive root of the positive operator.) Let $H=$ 
$H^{2}\left(C_{*}\right) \oplus \overline{\Delta L^{2}(C)}$, where the second summand denotes the $L^{2}(C)$ closure of $\left\{\Delta(t) g(t) \mid g \in L^{2}(C)\right\}$, and $M=\left\{(S(z) f(z), \Delta(t) f(t)) \mid f \in H^{2}(C)\right\} \subset H$. Then $M$ is invariant under the (unilateral) shift $U_{+}$in $H$ defined by $U_{+}(f, g)=\left(z f(z), e^{i t} g(t)\right)$, so $K=H \ominus M$ is invariant under $U_{+}^{*}$, where $U_{+}^{*}$ is the left-shift defined by $U_{+}^{*}(f, g)=\left(z^{-1}(f(z)-f(0)), e^{-i t} g(t)\right)$. We call $K$ the Sz.-Nagy-Foias space generated by $S$. Let $T$ denote the restricted right shift in $K$, i.e. the compression of $U_{+}$to $K$. Thus, for $(f, g) \in K, T(f, g)=P\left(z f, e^{2 t} g\right)$, where $P$ denotes projection onto $K$, and $T^{*}=\left.U_{*}^{*}\right|_{K}$. Note that if $S$ is inner, then $\Delta(t)=0$ a.e. and $K=H^{2}(C) \ominus S H^{2}(C)$.

Let $\widetilde{S}(z)$ be the analytic operator-valued function defined by $\widetilde{S}(z)=S(\bar{z})^{*}$, i.e. $\widetilde{S}(t)=S(-t)^{*}: C_{*} \rightarrow C$. Analogously to above, define $\widetilde{\Delta}(t): C_{*} \rightarrow C_{*}$ by $\widetilde{\Delta}(t)=\left(I-\widetilde{S}(t)^{*} \widetilde{S}(t)\right)^{1 / 2}, \widetilde{H}=H^{2}(C) \oplus \widetilde{\Delta L^{2}\left(C_{*}\right)}, \widetilde{M}=$ $\left\{(\widetilde{S} f, \widetilde{\Delta} f) \mid f \in H^{2}\left(C_{*}\right)\right\}, \widetilde{K}=\widetilde{H} \ominus \widetilde{M}$, and $\widetilde{T}=\widetilde{P} \widetilde{U}_{+} \mid \widetilde{K}$, where $\widetilde{U}_{+}$is the unilateral shift in $\widetilde{H}$ and $\widetilde{P}$ is projection onto $\widetilde{K}$. Note that $\widetilde{S}$ is inner if and only if $S$ is inner. (We use "inner" in the sense of [6], i.e. $S(t): C \rightarrow C_{*}$ is unitary a.e.; in the terminology of [8], this is called "inner from both sides".) The following is an extension of [4, Theorem 2.1].

THEOREM 1.1. The right shift $T$ on $K$ is unitarily equivalent to the left shift $\widetilde{T}^{*}$ on $\widetilde{K}$.

Proof. Let $L=L^{2}\left(C_{*}\right) \oplus \overline{\Delta L^{2}(C)}, \widetilde{L}=L^{2}(C) \oplus \overline{\widetilde{\Delta L^{2}\left(C_{*}\right)}}$, and consider $\tau: L \rightarrow \widetilde{L}$ defined by

$$
\begin{aligned}
\tau(f, \Delta g)= & e^{-i t}\left(S(-t)^{*} f(-t)+\Delta^{2}(-t) g(-t),\right. \\
& \widetilde{J}(t)(f(-t)-S(-t) g(-t))) .
\end{aligned}
$$

Then

$$
\begin{aligned}
\|\tau(f, \Delta g)\|_{\widetilde{L}}^{2}= & \left\|S(-t)^{*} f(-t)+\Delta^{2}(-t) g(-t)\right\|_{L^{2}(C)}^{2} \\
& +\|\widetilde{\Delta}(t)(f(-t)-S(-t) g(-t))\|_{L^{2}(C *)}^{2} \\
= & \|f(-t)\|^{2}+\left(\|g(-t)\|^{2}-\left\|S(-t)^{*} S(-t) g(-t)\right\|^{2}\right) \\
= & \|f(t)\|_{L^{2}\left(C_{*}\right)}^{2}+\|\Delta(t) g(t)\|_{L^{2}(C)}^{2}=\|(f, \Delta g)\|_{L}^{2},
\end{aligned}
$$

so $\tau$ extends to an isometry from $L$ to $\tilde{L}$. For $f \in L^{2}(C), g \in L^{2}\left(C_{*}\right)$, $(f, \Delta g)=\tau\left(\tau^{*}(f, \Delta g)\right)$, where $\tau^{*}(f, \Delta g)=e^{-i t}\left(S(t) f(-t)+\tilde{\Delta}^{2}(-t) g(-t)\right.$, $\left.\Delta(t)\left(f(-t)-S(t)^{*} g(-t)\right)\right)$, so $\tau$ is unitary.

We can decompose $L=K \oplus M \oplus K^{2}\left(C_{*}\right)$, where

$$
K^{2}\left(C_{*}\right)=\left\{(f, 0) \mid f \in L^{2}\left(C_{*}\right) \ominus H^{2}\left(C_{*}\right)\right\},
$$

and similarily $\widetilde{L}=\widetilde{K} \oplus \widetilde{M} \oplus K^{2}(C)$. It is easy to see that $\tau(M)=$ $K^{2}(C)$ and $\tau\left(K^{2}\left(C_{*}\right)\right)=\tilde{M}$, so therefore $\tau(K)=\widetilde{K}$. Hence, $\tau P=\widetilde{P} \tau$ (here we consider the domains of $P$ and $\widetilde{P}$ to be $L$ and $\widetilde{L}$ respectively), 
and $\tau U=\widetilde{U}^{*} \tau$, where $U$ and $\widetilde{U}$ are the bilateral shifts on $L$ and $\tilde{L}$. Thus, $\tau P U=\widetilde{P} \widetilde{U}^{*} \tau$, which implies $\tau T=\widetilde{T}^{*} \tau$ on $K$. Therefore, $\left.\tau\right|_{K}$, which we denote simply by $\tau$, is a unitary map from $K$ to $\widetilde{K}$ satisfying the theorem.

It is now easy to derive an explicit formula for $T$ will which be useful later on.

Corollary. For $(f, \Delta g) \in K$,

$$
T(f, \Delta g)=\left(z f(z)-S(z) Q(0), e^{i t} \Delta(t) g(t)-\Delta(t) Q(0)\right)
$$

where $Q(z)$ is the first component of $\tau(f, \Delta g)$.

Proof. This is obtained by computing

$$
\left(\tau^{*} \widetilde{T}^{*} \tau\right)(f, \Delta g) \text {. }
$$

If $F=(f, g) \in K$ and $\tau(F)=(Q, h)$, denote by $\left(\tau_{1} F\right)(z)$ the $C$ valued function $Q(z)$. We derive several technical lemmas needed later on.

Lemma 1.2. For $|w|<1, x \in C_{*}, y \in C$, let

$$
\begin{aligned}
k_{w, x, y}= & \left(\frac{I-S(z) S(w)^{*}}{1-z \bar{w}} x,-\frac{\Delta(t) S(w)^{*}}{1-e^{i t} \bar{w}} x\right) \\
& +\left(\frac{S(z)-S(\bar{w})}{z-\bar{w}} y, \frac{\Delta(t)}{e^{i t}-\bar{w}} y\right)
\end{aligned}
$$

Then $k_{w, x, y} \in K$ and

$$
\begin{aligned}
& P((x /(1-z \bar{w}), 0))=k_{w, x, 0}, \\
& P\left(\left(\frac{S(t)}{e^{i t}-\bar{w}} y, \frac{\Delta(t)}{e^{i t}-\bar{w}} y\right)\right)=k_{w, 0, y} .
\end{aligned}
$$

Proof. Note, for $(S f, \Delta f) \in M$,

$$
\begin{aligned}
\left(k_{w, x, 0},(S f, \Delta f)\right)= & \left(\frac{I-S(z) S(w)^{*}}{1-z \bar{w}} x, S(z) f(z)\right) \\
& -\left(\frac{\Delta(t)^{2} S(w)^{*}}{1-e^{i t} \bar{w}} x, f(t)\right)=0
\end{aligned}
$$

and hence $k_{w, x, 0} \in K$.

Similarly

$$
\begin{aligned}
& \left(k_{w, 0, y},(S f, \Delta f)\right) \\
& \quad=\left(\frac{S(z)-S(\bar{w})}{z-\bar{w}} y, S(z) f(z)\right)+\left(\frac{\Delta(t)^{2}}{e^{i t}-\bar{w}} y, f(t)\right)
\end{aligned}
$$




$$
\begin{aligned}
& =\left(\frac{S(t)^{*} S(\bar{w})}{e^{i t}-\bar{w}} y, f(t)\right)+\left(\frac{1}{e^{i t}-\bar{w}} y, f(t)\right) \\
& =0+0=0,
\end{aligned}
$$

since $f(t) \in H^{2}(C)$. Hence $k_{w, 0, y} \in K$.

Furthermore,

$$
\begin{aligned}
(x /(1 & -z \bar{w}), 0)-k_{w, x, 0} \\
& =\left(S(z) \frac{S(w)^{*}}{1-z \bar{w}} x, \Delta(t) \frac{S(w)^{*}}{1-e^{i t} \bar{w}} x\right) \in M
\end{aligned}
$$

and

$$
\begin{gathered}
\left(S(t) y /\left(e^{i t}-\bar{w}\right), \Delta(t) y /\left(e^{i t}-\bar{w}\right)\right)-k_{w, 0, y} \\
=\left(\frac{S(\bar{w})}{e^{i t}-\bar{w}} y, 0\right) \in(K \oplus M)^{\perp}
\end{gathered}
$$

All the assertions of the lemma follow.

LEMma 1.3. If $(f, g)=F \in K$, then

(i) $\left(F, k_{w, x, 0}\right)_{K}=(f(w), x)_{C *}$

(ii) $\left(F, k_{w, 0, y}\right)_{K}=\left(\left(\tau_{1} F\right)(w), y\right)_{C}$.

In particular

(iii) for $x, y \in C_{*}$, $\zeta$ and $\eta$ in $D$,

$$
\left(k_{\zeta, x, 0}, k_{\eta, y, 0}\right)_{K}=\left(\frac{I-S(\eta) S(\zeta)^{*}}{1-\eta \bar{\zeta}} x, y\right)_{C *}
$$

(iv) for $x, y \in C$,

$$
\left(k_{\zeta, 0, x}, k_{\eta, 0, y}\right)_{K}=\left(\frac{I-S(\bar{\eta})^{*} S(\bar{\zeta})}{1-\eta \bar{\zeta}} x, y\right)_{c}
$$

and

(v) for $x \in C, y \in C_{*}$,

$$
\left(k_{\zeta, 0, x}, k_{\eta, y, 0}\right)_{K}=\left(\frac{S(\eta)-S(\bar{\zeta})}{\eta-\bar{\zeta}} x, y\right)_{C_{*}} .
$$

Proof. For $(f, g) \in K, x \in C_{*}$, we have

$$
\begin{aligned}
(f(w), x) & =\left((f, g),\left(\frac{1}{1-z \bar{w}} x, 0\right)\right) \\
& =\left((f, g), P\left(\frac{1}{1-z \bar{w}} x, 0\right)\right) \\
& =\left((f, g), k_{w, x, 0}\right)
\end{aligned}
$$

by Lemma 1.2, proving (i). 
For (ii) note that

$$
\left(\tau_{1} F\right)(t)=e^{-i t}\left(S(-t)^{*} f(-t)+\Delta(-t) g(-t)\right)
$$

is in $H^{2}(C)$. Hence

$$
\begin{aligned}
\left(\left(\tau_{1} F\right)(w), y\right)_{C} & =\left(e^{-i t}\left(S(-t)^{*} f(-t)+\Delta(-t) g(-t)\right), \frac{1}{1-e^{i t} \bar{w}} y\right) \\
& =\left(S(t)^{*} f(t)+\Delta(t) g(t), \frac{1}{e^{2 t}-\bar{w}} y\right) \\
& =\left((f, g),\left(\frac{S(t)}{e^{i t}-\bar{w}} y, \frac{\Delta(t)}{e^{i t}-\bar{w}} y\right)\right) \\
& =\left((f, g), P\left(\frac{S(t)}{e^{i t}-\bar{w}} y, \frac{\Delta(t)}{e^{i t}-\bar{w}}\right)\right) \\
& =\left((f, g), k_{w, 0, y}\right)
\end{aligned}
$$

by Lemma 1.2, and (ii) follows.

A straight-forward computation gives

$$
\left(\tau_{1} k_{w, x, 0}\right)(z)=\frac{\widetilde{S}(z)-\widetilde{S}(\bar{w})}{z-\bar{w}} x
$$

and

$$
\left(\tau_{1} k_{w, 0, y}\right)(z)=\frac{I-\widetilde{S}(z) \widetilde{S}(w)^{*}}{1-z \bar{w}} y .
$$

Hence (iii)-(v) follows from (i) and (ii).

We note that if $F=(f, g) \in K$ is orthogonal to $k_{w, w, y}$ for all $w \in D, x \in C_{*}$ and $y \in C$, then $f=0$ and $\tau_{1} F=0$. From the formula for $\tau_{1}$, it follows that also $g=0$, and hence $F$ is the zero element of $K$. This implies that $\left\{k_{w, x, y} \mid w \in D, x \in C_{*}, y \in C\right\}$ spans a dense subset of $K$. This fact will make the formulas (iii)-(v) useful for computations later on.

The next lemma follows from the corollary to Theorem 1.1 and direct computations.

LEMMA 1.4. (i) $T k_{w, x, 0}=\bar{w}^{-1}\left(k_{w, x, 0}-k_{0, x, 0}\right), w \neq 0$.

(ii) $T k_{w, 0, y}=\bar{w} k_{w, 0, y}-k_{0, S(\bar{w}) y, 0}$.

(iii) $T^{*} k_{w, x, 0}=\bar{w} k_{w, x, 0}-k_{0,0, S(w) * x}$

(iv) $T^{*} k_{w, 0, y}=\bar{w}^{-1}\left(k_{w, 0, y}-k_{0,0, y}\right), w \neq 0$.

We wish to distinguish two subspaces of $K$ defined by

$$
\begin{aligned}
& k_{0}=\text { the closure of }\left\{k_{0, x, 0} \mid x \in C_{*}\right\} \\
& K_{0}=\text { the closure of }\left\{k_{0,0, y} \mid y \in C\right\} .
\end{aligned}
$$


Let us simplify the notation for this special case by writing $d_{x}$ for $k_{0, x, 0}$ and $D_{y}$ for $k_{0,0, y}$.

Lemma 1.5. Let $F=(f, g) \in K$. Then

(i ) $T^{*} F=\left(z^{-1} f(z), e^{-i t} g(t)\right)$ if and only if $F \perp k_{0}$.

(ii) $T F=\left(z f(z), e^{i t} g(t)\right)$ if and only if $F \perp K_{0}$.

Proof. (i) holds if and only if $f(0)=0$ which holds if and only if $F \perp k_{0}$ by Lemma 1.3 (i). By the corollary to Theorem 1.1, (ii) follows similarly, using Lemma 1.3 (ii).

Lemma 1.6. Let $P_{k_{0}}$ and $P_{K_{0}}$ denote the orthogonal projection onto $k_{0}$ and $K_{0}$ respectively. Then $P_{k_{0}} F=d_{x}$, where

$$
x=\left(I-S(0) S(0)^{*}\right)^{-1} f(0)
$$

and $P_{K_{0}} F=D_{y}$, where $y=\left(I-S(0)^{*} S(0)\right)^{-1}\left(\tau_{1} F\right)(0)$. (Note since $S(z)$ is a pure contractive function, $x$ and $y$ are well-defined for $F$ in some dense subset of $K$.)

Proof. The map $e_{1}$ densely defined by $e_{1}: x \rightarrow d_{\left(I-S(0) S(0)^{*}\right)-1 / 2 x}$ is an isometry (using Lemma 1.3iii) of $C_{*}$ into $K$ with range equal to $k_{0}$, and with adjoint given by $e_{1}^{*}: f \rightarrow\left(I-S(0) S(0)^{*}\right)^{-1 / 2} f(0)$ (using Lemma 1.3i). The formula for $P_{k_{0}}$ follows by computing $e_{1} e_{1}^{*}$. The formula for $P_{K_{0}}$ follows similarly.

\section{The perturbations.}

Definition 2.1. Let $A: C \rightarrow C_{*}$ be a bounded linear map. We define $Z(A)$ to be the unique bounded linear map on $K$ such that

$$
Z(A) F=\left\{\begin{array}{lll}
T F & \text { if } \quad F \perp K_{0} \\
d_{A y} & \text { if } \quad F=D_{y} .
\end{array}\right.
$$

REMARK 2.2. It follows that $Z(A)^{*}$ is given by

$$
Z(A)^{*} F=\left\{\begin{aligned}
& T^{*} F \text { if } F \perp k_{0} \\
& D_{y} \text { if } F=d_{x}, \text { where } y \\
&=\left(I-S(0)^{*} S(0)\right)^{-1} A^{*}\left(I-S(0) S(0)^{*}\right) x .
\end{aligned}\right.
$$

We note that $T=Z\left(-S(0)\right.$ ) (by Lemma 1.3), and that $Z(A)^{*} d_{x}=$ $D_{A^{*} x}$ if and only if

$$
A S(0)^{*} S(0)=S(0) S(0)^{*} A .
$$

THEOREM 2.3. (i) $Z(A)$ is a contraction if and only if 


$$
A^{*}(I-S(0) * S(0)) A \leqq\left(I-S(0) S(0)^{*}\right) .
$$

(ii) $Z(A)$ is unitary if and only if $A=\left(I-S(0) S(0)^{*}\right)^{-1 / 2} V(I-$ $\left.S(0)^{*} S(0)\right)^{1 / 2}$ for some unitary $V$.

(iii) If $A$ satisfies condition (1), then $Z(A)$ is a contraction if and only if $\|A\| \leqq 1$ and $Z(A)$ is unitary if and only if $A$ is unitary.

Proof. (i) Since $Z(A)$ maps $K_{0}^{\perp}$ isometrically onto $k_{0}^{\perp}$ and sends $K_{0}$ onto $k_{0}, Z(A)$ is a contraction if and only if it is contractive on $K_{0}$. By Lemma 1.3, this holds precisely when $\|A y\|^{2}-\left\|S(0)^{*} A y\right\|^{2} \leqq$ $\|y\|^{2}-\|S(0) y\|^{2}$ for all $y \in C$, but this is clearly equivalent to (2).

(ii) As above, $Z(A)$ is isometric precisely when equality holds in (2). By [5, Theorem 1.7(i)], this holds if and only if $A=(I-$ $\left.S(0) S(0)^{*}\right)^{-1 / 2} V(I-S(0) * S(0))^{1 / 2}$ for some isometry $V$. By Lemma 1.3, $Z(A)^{*}$ is isometric if and only if $\left(I-S(0) S(0)^{*}\right)=\left(I-S(0) S(0)^{*}\right)^{1 / 2} V V^{*}(I-$ $\left.S(0) S(0)^{*}\right)^{1 / 2}$, which holds if and only if $V V^{*}=I$, so $V$ must be unitary.

(iii) If (1) holds, then (2) reduces to $\left(A^{*} A\right)\left(I-S(0)^{*} S(0)\right) \leqq$ $\left(I-S(0)^{*} S(0)\right)$, which, using (1) again, holds if and only if $A^{*} A \leqq$ $I$, i.e. $\|A\| \leqq 1$. In the second case, (1) implies that $A=V$.

REMARK 2.4. In [5], it was claimed that (1) was a necessary condition for $Z(A)$ to be a contraction. Clearly, if $A$ is bounded, then $Z(\lambda A)$ will be a contraction for all sufficiently small scalars $\lambda$. There is also an error in Theorem 1.7(iii) in [5], which states that if $P$ and $Q$ are unitarily equivalent strictly positive operators and $X$ is a solution of $P \geqq X^{*} Q X$ and $Q \geqq X P X^{*}$, then $X$ is a contraction such that $X P=Q X$. The matrices

$$
P=\left[\begin{array}{ll}
1 & 0 \\
0 & 2
\end{array}\right], Q=\left[\begin{array}{ll}
2 & 0 \\
0 & 1
\end{array}\right], X=\frac{1}{\sqrt{2}}\left[\begin{array}{ll}
1 & 0 \\
0 & 0
\end{array}\right]
$$

provide a counterexample.

3. Characteristic functions and spectra. The Sz.-Nagy-Foiaș model theory for contractions assigns to each contraction $T$ on a Hilbert space $H$ the triple $\left\{\mathscr{D}_{T}, \mathscr{D}_{T^{*}}, \Theta_{T}(\lambda)\right\}$ where $D_{T}=\left(I-T^{*} T\right)^{1 / 2}$, $D_{T^{*}}=\left(I-T T^{*}\right)^{1 / 2}, \mathscr{D}_{T}=\overline{D_{T} H}, \mathscr{D}_{T^{*}}=\overline{D_{T^{*}} H}$, and $\Theta_{T}(\lambda)=\left[-T+\lambda D_{T^{*}}(I-\right.$ $\left.\left.\lambda T^{*}\right)^{-1} D_{T}\right]\left.\right|_{\mathscr{D}_{T}}$ is an analytic operator-valued function whose values are contractions from $\mathscr{D}_{T}$ to $\mathscr{D}_{T^{*}}$, the defect spaces of $T$. (This holds since $T D_{T}=D_{T^{*}} T$.) We call this triple the characteristic function of $T$, and if $T$ is completely nonunitary (c.n.u.), i.e. there is no reducing subspace on which $T$ is unitary, then $T$ is unitarily equivalent to the adjoint of the restricted shift on the Sz.-Nagy-Foias, space generated by its characteristic function [8, p. 248]. In most 
cases, one is unable to get any "concrete" information from this representation for a specific operator because of computational difficulties involved in simplifying the form of the characteristic function. However, if $A$ satisfies (1), then we can apply Fuhrmann's proof [5, p. 169-172] verbatim to get the following two theorems.

THEOREM 3.1. If $A$ is a strict contraction satisfying (1), then $Z(A)$ is a c.n.u. contraction on $K$ with characteristic function $\left\{K_{0}, k_{0}, \Theta_{Z(A)}(z)\right\}$, where $\Theta_{Z(A)}(z)$ is given by $\Theta_{Z(A)}(z) D_{y}=d_{G(z) y}$ where

$$
\begin{aligned}
& \left(I-S(0) S(0)^{*}\right)^{1 / 2} G(z)\left(I-S(0)^{*} S(0)\right)^{-1 / 2} \\
& \quad=\left(I-A A^{*}\right)^{1 / 2}\left(I-\Gamma(z) A^{*}\right)^{-1}(\Gamma(z)-A)\left(I-A^{*} A\right)^{-1 / 2}
\end{aligned}
$$

and

$$
\Gamma(z)=\left(I-S(0) S\left(0^{*}\right)\right)^{1 / 2}\left(I-S(z) S(0)^{*}\right)^{-1}(S(z)-S(0))\left(I-S(0)^{*} S(0)\right)^{-1 / 2} .
$$

Note that the above are matrix fractional linear transformations.

We call an open arc $\gamma$ of the unit circle regular for $S(z)$ if $S(z)$ has analytic continuation over $\gamma$ and for all $\lambda \in \gamma, S(\lambda)$ is unitary. Let $\sigma(T)$ and $\sigma(Z(A)$ ) denote the spectrum of $T$ and $Z(A)$ respectively. Recall [8, Theorem VI, 4.1] that $\sigma(T)=\{|z|<1 \mid S(z)$ is not boundedly invertible $\} \cup\{|\lambda|=1 \mid \lambda$ lies on no regular are of $S\}$.

THEOREM 3.2. Under the assumptions of 3.1, (i) $\sigma(Z(A))=\{|\lambda|=$ $1 \mid \lambda$ lies on no regular arc of $S\} \cup\{|z|<1 \mid(\Gamma(z)-A)$ is not boundedly invertible\}.

(ii) $Z(A)^{n}$ and $Z(A)^{* n}$ both converge to zero in the strong operator topology if and only if $S(Z)$ is inner.

REMARK 3.3. (a) If $A$ is a strict contraction not satisfying (1), $\Theta_{Z(A)}(z)$ as defined above still has an interpretation as a characteristic function. (b) Note that $W \equiv Z(0)$ is the completely nonunitary partial isometry with initial space $K_{0}$ and final space $k_{0}$ and agreeing with $T$ on $K_{0}$ With this choice of $A,(1)$ is satisfied and Theorem 3.1 says $\Theta_{Z(0)}(z) D_{y}=d_{(I-S(0) S(0) *)-1 / 2 \Gamma(z)(I-S(0) * S(0) 1 / 2 y}$. It is not difficult to see that $\Theta_{Z(0)}(z)$ coincides (see [8] page 192 for definition) with $\Gamma(z)$ : $C \rightarrow C_{*}$. Hence the partial isometry $W$ can be represented as the compressed right shift $T^{\prime}$ on the Sz.-Nagy-Foias space $K^{\prime}$ associated with $\Gamma(z)$ rather than with $S(z)$. (c) For $A$ a contraction from $C$ to $C_{*}$, let $Z^{\prime}(A)$ be the associated perturbation of $T^{\prime}$ in $K^{\prime}$. Since $\Gamma(0)=0$, (1) is satisfied for any $A$. In particular, for $A=-S(0)$, Theorem 3.1 gives $\Theta_{Z^{\prime}(-S(0))}(z) D_{y}^{\prime}=d_{S(z) y}^{\prime}$, and hence $\Theta_{Z^{\prime}(-S(0))}(z)$ coincides with $S(z)$. Hence the operator $T$ on $K$ is unitarily equivalent to 
$Z^{\prime}(-S(0))$ on $K^{\prime}$. It is then clear that the formula above for $\Theta_{Z(A)}$ ( $A$ not necessarily satisfying (1)), interpreted for $D_{y}$ and $d_{z}$ in $K^{\prime}$, gives the characteristic function for $Z^{\prime}(A)$. In this sense, it is perhaps more natural to study perturbations of $Z^{\prime}(-S(0))$ on $K^{\prime}$ rather than of $T$ on $K$. It is now seen that (1) is the condition that $Z(A)$ and $Z^{\prime}(A)$ be unitarily equivalent.

4. Unitary perturbations. Since the characteristic function of a unitary map is zero, the above method fails totally when $Z(A)$ is unitary. However, when $A$ satisfies (1) we can still get spectral information about $Z(A)$ by adapting techniques of D. N. Clark [2] to a more general setting. We begin with two technical lemmas.

Lemma 4.1. If $A$ is unitary and satisfies (1), then $\alpha=\alpha_{A}=$ $-\left(I+S(0) A^{*}\right)\left(S(0)^{*}+A^{*}\right)^{-1}$ is unitary from $C$ to $C_{*}$.

Proof. $\alpha$ is a priori defined on some dense set $D_{1} \subset C$ since $S(0)^{*}$ is a pure contraction and $A^{*}$ is unitary. (1) implies that

$$
\left(I+A S(0)^{*}\right)\left(I+S(0) A^{*}\right)\left(S(0)^{*}+A^{*}\right)^{-1}=(S(0)+A) .
$$

Thus, for $x \in D_{1},(\alpha x, \alpha x)=\left(\left(S(0)^{*}+A^{*}\right)^{-1} x,(S(0)+A) x\right)=(x, x)$, so $\alpha$ can be extended to an isometry on $C$. Similarly, $\alpha^{*}$ is an isometry on $C_{*}$ so $\alpha$ is unitary.

Note that in fact, (1) is also necessary for $\alpha$ to be unitary, and $\alpha_{A}$ determines $A$ by $A^{*}=-(\alpha+S(0))^{-1}\left(\alpha S(0)^{*}+I\right)$. Also, we have $(\alpha+S(0))^{-1}=-\left(S(0)^{*}+A^{*}\right)\left(I-S(0) S(0)^{*}\right)^{-1}$; our $\alpha$ corresponds to $-\alpha$ used in [2].

LeMma 4.2. For $F=(f, g) \in K$,

(i ) $(Z(A)-T)(F)=k_{0, x, 0}$ where $x=-\left(\alpha^{*}+S(0)^{*}\right)^{-1}\left(\tau_{1} F\right)(0)$

(ii) $\left(Z(A)^{*}-T\right)(F)=k_{0,0, y}$ where $y=-(\alpha+S(0))^{-1} f(0)$

Proof. Since $Z(A)=T\left(I-P_{K_{0}}\right)+Z(A) P_{K_{0}}$, we obtain

$$
\begin{aligned}
(Z(A)-T)(F) & =d_{(S(0)+A)(I-S(0) * S(0))-1\left(\tau_{1} F\right)(0)} \\
& =d_{x},
\end{aligned}
$$

with $x$ as in (i).

(ii) follows similarly.

For $|z|<1$, define $\varphi(z): C_{*} \rightarrow C_{*}$ by $\varphi(z)=\left(I-S(z) \alpha^{*}\right)\left(I+S(z) \alpha^{*}\right)^{-1}$. Then straight-forward calculation gives

$$
\varphi(\zeta)+\varphi(\eta)^{*}=2\left(I+S(\zeta) \alpha^{*}\right)^{-1}\left(I-S(\zeta) S(\eta)^{*}\right)\left(I+\alpha S(\eta)^{*}\right)^{-1}
$$

and hence (let $z=\zeta=\eta$ ) $\varphi(z)$ has nonnegative real part for $|z|<1$. By the operator-valued version of the Herglotz theorem, there exists 
a non-negative operator-valued measure $\mu$ on $[0,2 \pi]$ such that $\varphi(z)=$ $\int_{0}^{2 \pi}\left(e^{i \theta}+z\right)\left(e^{i \theta}-z\right)^{-1} d \mu(\theta)$.

Thus

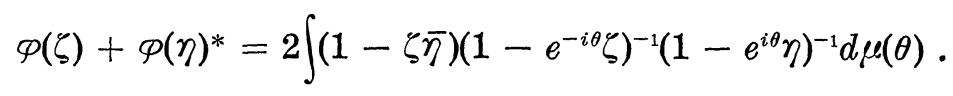

Comparing (3) and (4) yields

$$
\frac{I-S(\zeta) S(\eta)^{*}}{1-\zeta \bar{\eta}}=\int \frac{I+S(\zeta) \alpha^{*}}{1-e^{-i \theta} \zeta} d \mu(\theta) \frac{I+\alpha S(\eta)^{*}}{1-e^{i \theta} \bar{\eta}}
$$

Similar computations give

$$
\begin{aligned}
\frac{S(\zeta)-S(\bar{\eta})}{\zeta-\bar{\eta}} & =-\frac{1}{2}\left(I+S(\zeta) \alpha^{*}\right)\left(\frac{\varphi(\zeta)-\varphi(\bar{\eta})}{\zeta-\bar{\eta}}\right)\left(I+S(\bar{\eta}) \alpha^{*}\right) \alpha \\
& =-\int \frac{I+S(\zeta) \alpha^{*}}{1-e^{-i \theta} \zeta} d \mu(\theta) \frac{I+S(\bar{\eta}) \alpha^{*}}{e^{i \theta}-\bar{\eta}} \alpha
\end{aligned}
$$

and

$$
\frac{I-\widetilde{S}(\zeta) \widetilde{S}(\eta)^{*}}{1-\zeta \bar{\eta}}=\int_{0}^{2 \pi} \alpha^{*} \frac{I+\alpha S(\bar{\zeta})^{*}}{e^{-i \theta}-\zeta} d \mu(\theta) \frac{I+S(\bar{\eta}) \alpha^{*}}{e^{i \theta}-\bar{\eta}} \alpha
$$

We define Hilbert space $L^{2}(\mu)$ as in Shulman [7]. For $f=x_{1} \chi_{E_{1}}+$ $\cdots+x_{n} \chi_{E_{n}}$ a simple $C_{*}$-valued function, where $\chi_{E_{1}}, \cdots, \chi_{E_{n}}$ are characteristic functions of disjoint Borel sets and $x_{1}, \cdots, x_{n}$ are corresponding elements of $C_{*}$ define

$$
\|f\|_{\mu}^{2}=\int(d \mu(t) f(t), f(t))=\left(\mu\left(E_{1}\right) x_{1}, x_{1}\right)+\cdots+\left(\mu\left(E_{n}\right) x_{n}, x_{n}\right) .
$$

This does not depend on the representation of $f(t)$ in terms of characteristic functions. Let $\mathscr{A}=\left\{f(t):[0,2 \pi] \rightarrow C_{*} \mid f\right.$ is Borel measurable, $\int\|f(t)\|^{2} d(u(t) x, x)<\infty$ for all $x \in C_{*}$, the range of $f(t)$ is contained in a finite dimensional subspace of $\left.C_{*}\right\}$. For $f \in \mathscr{A}$ let $e_{1}, e_{2}, \cdots, e_{k}$ be a basis for the smallest subspace which contains the range of $f(t)$, and define

$$
\alpha(f, t)=\left(\mu(t) e_{1}, e_{1}\right)+\cdots+\left(\mu(t) e_{k}, e_{k}\right) .
$$

The definition is independent of the choice of basis for this subspace, and $\|f\|_{\mu}^{2} \leqq \int\|f(t)\|^{2} d \alpha(f, t)$ whenever $f$ is a simple function. For $f \in \mathscr{A}$, there is a sequence of simple functions $\left\{f_{n}(t)\right\}$ such that the range of $f_{n}(t)$ is contained in the range of $f(t)$ for $n=1,2, \cdots$, and such that $\int\left\|f_{n}(t)-f(t)\right\|^{2} d \alpha(f, t) \rightarrow 0$ as $n \rightarrow \infty$. We can define $\|f(t)\|_{\mu}^{2}$ unambiguously as 


$$
\|f\|_{\mu}^{2}=\lim _{n \rightarrow \infty}\left\|f_{n}\right\|_{\mu}^{2}
$$

By $L^{2}(\mu)$ is meant the Hilbert space completion of the inner product space of equivalence classes of functions with finite-dimensional range in $\mu$-norm. The definition of $L^{2}(\mu)$ is such that explicit formulas can be written only for an element associated with the equivalence class of an element of $\mathscr{A}$. This, however, causes no difficulties for our purposes. It is clear, for example, that the transformation $h(t) \rightarrow$ $e^{i t} h(t)$ is unitary in $L^{2}(\mu)$, with spectrum equal to supp $(\mu)$ (the complement of the largest open set on which $\mu$ is zero).

We are now in position to define a unitary transformation of $K$ onto $L^{2}(\mu)$ which transforms the operator $Z(A)$ on $K$ to the operator of multiplication on $e^{i t}$ on $L^{2}(\mu)$.

THEOREM 4.3. Define $V$ on elements in $K$ of the form $k_{\xi, x, y}$ by

$$
V\left(k_{\zeta, x, y}\right)=\frac{I+\alpha S(\zeta)^{*}}{1-e^{i t \bar{\zeta}}} x-\frac{I+S(\bar{\zeta}) \alpha^{*}}{e^{i t}-\bar{\zeta}} \alpha y .
$$

Then $V$ is well-defined and extends uniquely to a unitary transformation (also $V$ ) of $K$ onto $L^{2}(\mu)$ such that $V Z(A)=e^{i t} V$.

Proof. We first check that $V$ is an isometry on those vectors where it is defined. Note, for $x, y \in C_{*}$,

$$
\begin{aligned}
\left(\tilde{k}_{\eta, y, 0}, k_{\zeta, x, 0}\right)_{K} & =\left(\frac{I-S(\zeta) S(\eta)^{*}}{1-\bar{\eta} \zeta} y, x\right)_{c_{*}} \\
& =\left(\int \frac{I+S(\zeta) \alpha^{*}}{1-e^{-i t \zeta}} d \mu(t) \frac{I+\alpha S(\eta)^{*}}{1-e^{i t} \eta} y, x\right)_{c_{*}} \text { by } \\
& =\left(\frac{I+\alpha S(\eta)^{*}}{1-e^{2 t} \bar{\eta}} y, \frac{I+\alpha S(\zeta)^{*}}{1-e^{i t \bar{\zeta}}} x\right)_{L^{2(\mu)}} \\
& =\left(V k_{\eta, y, 0}, V k_{\zeta, x, 0}\right)_{L^{2}(\mu)} .
\end{aligned}
$$

Also, for $x, y \in C$,

$$
\begin{aligned}
\left(k_{\eta, 0, y}, k_{\zeta, 0, x}\right)_{K^{*}} & =\left(\frac{I-S(\bar{\zeta})^{*} S(\bar{\eta})}{1-\bar{\eta} \zeta} y, x\right)_{C} \\
& =\left(\int \alpha^{*} \frac{I+\alpha S(\bar{\zeta})^{*}}{e^{-i t}-\zeta} d \mu(t) \frac{I+S(\bar{\eta}) \alpha^{*}}{e^{i t}-\bar{\eta}} \alpha y, x\right)_{C} \\
& =\left(\frac{I+S(\bar{\eta}) \alpha^{*}}{e^{i t}-\bar{\eta}} \alpha y, \frac{I+S(\bar{\zeta}) \alpha^{*}}{e^{i t}-\bar{\zeta}} \alpha x\right)_{L^{2}(t)} \\
& =\left(V k_{\eta, 0, y}, V k_{\zeta, 0, x}\right)_{L^{2}(\mu)}
\end{aligned}
$$

and finally, for $x \in C_{*}$ and $y \in C$, 


$$
\begin{aligned}
\left(k_{\eta, 0, y}, k_{\zeta, x, 0}\right)_{K} & =\left(\frac{S(\zeta)-S(\bar{\eta})}{\zeta-\bar{\eta}} y, x\right)_{C_{*}} \\
& =\left(-\int \frac{I+S(\zeta) \alpha^{*}}{1-e^{-i t \zeta}} d \mu(t) \frac{I+S(\bar{\eta}) \alpha^{*}}{e^{i t}-\bar{\eta}} \alpha y, x\right)_{c_{*}} \text { by } \\
& =\left(V k_{\eta, 0, y}, V k_{\zeta, x, 0}\right)_{L^{2}(\mu)} .
\end{aligned}
$$

Hence $V$ is isometric (and hence also well-defined) on its domain. Since elements of the form $k_{\eta, x, y}$ span a dense set in $K, V$ extends by linearity and continuity to be an isometry of $K$ into $L^{2}(\mu)$. Since the range of $V$ contains all elements of the form $x /\left(1-e^{i t} \bar{w}\right)$ and $x /\left(e^{i t}-\bar{w}\right)$ for $x \in C_{*}$ and $|w|<1$, it follows that $V$ is onto $L^{2}(\mu)$.

It remains to show $V Z(A)=e^{i t} V$. By Lemmas 1.4 and 4.2,

$$
\begin{aligned}
Z(A)\left(k_{w, x, 0}\right)= & \bar{w}^{-1} k_{w, x, 0}-\bar{w}^{-1} k_{0, x, 0} \\
& +\bar{w}^{-1} k_{0,\left(\alpha^{*}+S(0) *\right)^{-1}\left(S(0) *-S(w)^{*} x, 0\right.} \\
= & \bar{w}^{-1}\left(k_{w, x, 0}-k_{0,\left(\alpha^{*}+S(0)^{*}\right)^{-1}\left(\alpha^{*}+S(w)\right) x, 0}\right)
\end{aligned}
$$

and hence

$$
\begin{aligned}
V Z(A) k_{w, x, 0} & =\bar{w}^{-1}\left(1-e^{i t} \bar{w}\right)^{-1}\left(I+\alpha S(w)^{*}\right) x-\bar{w}^{-1}\left(I+\alpha S(w)^{*}\right) x \\
& =\bar{w}^{-1}\left[\left(1-e^{i t} \bar{w}\right)^{-1}-1\right]\left(I+\alpha S(w)^{*}\right) x \\
& =e^{i t} \frac{I+\alpha S(w)^{*}}{1-e^{i t} \bar{w}} x=e^{i t} V k_{w, x, 0} .
\end{aligned}
$$

Similarly

$$
\begin{aligned}
Z(A) k_{w, 0, y}= & \bar{w} k_{w, 0, y}-k_{0, S(\bar{w}) y, 0} \\
& -k_{0,\left(\alpha^{*}+S(0) *\right)-1(I-S(0) * S(\bar{w})) y, 0} \\
= & \bar{w} k_{w, 0, y}-k_{0,\left(\alpha^{*}+S(0) *\right)-1\left(I+\alpha^{*} S(\bar{w})\right) y, 0} .
\end{aligned}
$$

So

$$
\begin{aligned}
V Z(A) k_{w, 0, y} & =-\bar{w}\left(e^{i t}-\bar{w}\right)^{-1}\left(I+S(\bar{w}) \alpha^{*}\right) \alpha y-\left(I+S(\bar{w}) \alpha^{*}\right) \alpha y \\
& =-e^{i t} \frac{I+S(\bar{w}) \alpha^{*}}{e^{i t}-\bar{w}} \alpha y \\
& =e^{i t} V k_{w, 0, y} .
\end{aligned}
$$

The theorem follows.

We note the following inversion formula for $V$.

TheOREM 4.4. Let $V^{*}: L^{2} \rightarrow K$ be defined, for $F$ in $\mathscr{A}$, by $V^{*} F=$ $\left(W_{1} F, W_{2} F\right)$ where $\left(W_{1} F\right)(z)=\left(I+S(z) \alpha^{*}\right) \int\left(1-e^{-i t} z\right) d \mu(t) F(t)$ and $\left(W_{2} F^{\prime}\right)(t)=\lim _{r \rightarrow 1}\left(I-S\left(r e^{i t}\right)^{*} S\left(r e^{i t}\right)\right)^{-1 / 2}$. 


$$
\begin{aligned}
\left(\alpha^{*}+\right. & \left.S\left(r e^{i t}\right)\right)^{*} \int\left(e^{2(t-\theta)}-r\right)^{-1} d \mu(\theta) F(\theta) \\
& -\int\left(S\left(r e^{i t}\right)^{*}-S\left(r e^{i t}\right)^{*} S\left(r e^{i t}\right) \alpha^{*}\right)\left(1-r e^{i(t-\theta)}\right)^{-1} d \mu(\theta) F(\theta) .
\end{aligned}
$$

Then $V^{*}$ is the adjoint of $V$ defined in Theorem 4.3.

Proof. To obtain $W_{1}$, rewrite equation (5) substituting $z$ for $\zeta$ and noting that

$$
\begin{aligned}
V k_{\eta, x, 0} & =\frac{I+\alpha S(\eta)^{*}}{1-e^{i t} \bar{\eta}} x \text { to obtain } \\
\frac{I-S(z) S(\eta)^{*}}{1-\zeta \bar{\eta}} x & =\int \frac{I+S(z) \alpha^{*}}{1-e^{-\imath t} z} d \mu(t)\left(V k_{\eta, x, 0}\right)(t) .
\end{aligned}
$$

Similarly, using equation (6),

$$
\frac{S(z)-S(\bar{\eta})}{z-\bar{\eta}} y=\int \frac{I+S(z) \alpha^{*}}{1-e^{-i t} z} d \mu(t)\left(V k_{\eta, 0, y}\right)(t) .
$$

This proves the correctness of the formula for $W_{1}$ for all $F$ of the form $V k_{r, x, y}$, and hence by approximation for all $F \in \mathscr{X}$. To obtain the formula for $W_{2}$, we first find a formula for $\left(\tau_{1} V^{*} F\right)(z)$. By an argument dual to that above, we find

$$
\left(\tau_{1} V^{*} F\right)(z)=-\alpha^{*}\left(I+\alpha S(\bar{z})^{*}\right) \int\left(e^{-\imath t}-z\right)^{-1} d \iota(t) F(t) .
$$

The formula for $W_{2}$ is then obtained by using the explicit formulas for $\tau$ and $\tau^{*}$ in Theorem 1.1 .

THEOREM 4.5. Let $A$ be unitary and satisfy (1). Then $\sigma(Z(A))=$ $\{|\lambda|=1 \mid \lambda$ lies on no regular arc of $S\} \cup\{|\lambda|=1 \mid \lambda$ lies on a regular arc of $S$ but $\left(I+S(\lambda) \alpha^{*}\right)$ is not boundedly invertible\}.

Proof. Since $Z(A)$ has a representation as multiplication by $e^{i \theta}$ on $L^{2}(\mu)$, we have $\sigma(Z(A))=\operatorname{supp}(\mu)$, the complement of the largest open set on which $\mu$ is zero. By the integral representation of $p$, we see that the complement of $\operatorname{supp}(\mu)$ is the set of $\lambda$ at which $\varphi(z)$ has analytic continuation with $\operatorname{Re} \varphi(\lambda)=0$. Since $\varphi(z)=$ $\left(I-S(z) \alpha^{*}\right)\left(I+S(z) \alpha^{*}\right)^{-1}$, we have $(I+\phi(z))=2\left(I+S(z) \alpha^{*}\right)^{-1}$ and $S(z)=(I-\varphi(z))(I+\varphi(z))^{-1} \alpha$.

Now, suppose $\varphi(z)$ has continuation at $\lambda$ and $\operatorname{Re} \varphi(\lambda)=0$. Then $(I+\mathcal{P}(\lambda))$ is boundedly invertible, and hence $(I+\mathcal{P}(z))^{-1}$ extends to an analytic function in a neighborhood of $\lambda$. Thus, $S(z)$ has analytic continuation at $\lambda$ and $\left(I+S(\lambda) \alpha^{*}\right)$ is boundedly invertible; since $\operatorname{Re} \varphi(\lambda)=0, S(\lambda)$ is unitary. Conversely, suppose $S(z)$ has analytic 
continuation at $\lambda,\left(I+S(\lambda) \alpha^{*}\right)$ is boundedly invertible, and $S(\lambda)$ is unitary. Then $\left(I+S(z) \alpha^{*}\right)^{-1}$ is analytic in some neighborhood of $\lambda$, so $\varphi(z)$ has analytic continuation at $\lambda$; since $S(\lambda)$ is unitary, $\operatorname{Re} \varphi(\lambda)=0$. By taking complements, the theorem now follows.

Since $\left(I+S(\lambda) \alpha^{*}\right)=\left[\left(I+S(0) A^{*}\right)-S(\lambda)\left(S(0)^{*}+A^{*}\right)\right]\left(I+S(0) A^{*}\right)^{-1}$, we see that $\left(I+S(\lambda) \alpha^{*}\right)$ is boundedly invertible if and only if $B(\lambda)=-\left[\left(I+S(0) A^{*}\right)-S(\lambda)\left(S(0)^{*}+A^{*}\right)\right]$ is boundedly invertible. With $\Gamma$ as in Theorem 3.1, we have, since $A$ satisfies $(1),(\Gamma(\lambda)-A)=$ $\left(I-S(0) S(0)^{*}\right)^{1 / 2}\left(I-S(\lambda) S(0)^{*}\right)^{-1} B(\lambda) A\left(I-S(0)^{*} S(0)\right)^{-1 / 2}$. Thus, $(\Gamma(\lambda)-A)$ is invertible, but not necessarily boundedly, if and only if $B(\lambda)$ is invertible. Since boundedness follows immediately in the finitedimensional case, we have the following generalization of [5, Theorem $3.6]$ to the case of general analytic contractions $S(z)$.

Corollary 4.6. If $A$ is unitary on $C, C$ finite-dimensional, and $A$ satisfies (1), then $\sigma(Z(A))=\{|\lambda|=1 \mid \lambda$ lies on no regular arc of $S\} \cup\{|\lambda|=1 \mid \lambda$ lies on a regular arc for $S$ but $(\Gamma(\lambda)-A)$ is not invertible\}.

In the finite-dimensional case, $Z(A)$ is a compact perturbation of $T$. Hence by the known spectral behavior of $T$ and Weyl's theorem, $\{|\lambda|=1 \mid \lambda$ lies on a regular arc for $S$ but $\Gamma(\lambda)-A$ is not invertible $\}$ must be eigenvalues for $Z(A)$.

We can also adapt Fuhrmann's calculations [5, page 174] to determine eigenvalues in our more general setting.

THEOREM 4.7. If $A$ is unitary and satisfies (1), and $\lambda$ lies on a regular arc for $S$, then $\lambda$ is an eigenvalue for $Z(A)$ if and only if the range of $\Gamma(\lambda)-A$ is not dense in $C_{*}$.

REMARK 4.8 If $A$ does not satisfy (1), all of the above results apply to $Z^{\prime}(A)$, as in Remark 3.3. Also, we still have from Theorem 2.3 that $A=\left(I-S(0) S(0)^{*}\right)^{-1 / 2} V\left(I-S(0)^{*} S(0)\right)^{1 / 2}$ for some unitary $V$. This implies that $\widetilde{\alpha}_{A}=\tilde{\alpha}=\left(A^{*}+S(0)^{*}\right)^{-1}\left(I+A^{*} S(0)\right)$ is unitary. (Note that if $A$ satisfies (1), then $\tilde{\alpha}=\alpha$ used above.) In this case, the results of $\S 4$ still hold with $\tilde{\alpha}$ in place of $\alpha$.

\section{REFERENCES}

1. L. de Branges and J. Rovnyak, Canonical Models in Quantum Scattering Theory, Perturbation theory and its applications in quantum mechanics, Wiley, New York, (1966), 295-391.

2. D. N. Clark, One dimensional perturbations of restricted shifts, J. Analyse Math., 25 (1972), 169-191.

3. —_, Extending Fourier transforms into Sz.-Nagy-Foiaș spaces, Bull. Amer. Math. Soc., 78 (1972), 65-67. 
4. P. A. Fuhrmann, On the corona theorem and its application to spectral problems in Hilbert space, Trans. Amer. Math. Soc., 132 (1968), 55-66.

5. P. A. Fuhrmann, On a class of finite dimensional contractive perturbations of restricted shifts of finite multiplicity, Israel J., Math., 16 (1973), 162-175.

6. H. Helson, Lectures on Invariant Subspaces, Academic Press, New York, 1964.

7. L. Shulman, Perturbations of unitary transformations, J. Math. Anal. and Appl., 28 (1969), 231-254.

8. B. Sz.-Nagy and C. Foiaș, Harmonic Analysis of Operators in Hilbert Space, NorthHolland Publishing Co., 1970.

Received December 4, 1975 and in revised form February 11, 1976. This paper contains some results from the first author's dissertation written under the direction of Marvin Rosenblum at the University of Virginia. The second author was partially supported by NSF Grant GP-38265. Portions of this paper were presented at the International Symposium of Operatory Theory and Network Systems, Montreal, Canada, August 1975.

Virginia Polytechnic Institute and State University

NORTHWESTERN UNIVERSITY

AND

ILLINOIS INSTITUTE OF TECHNOLOGY 



\title{
PACIFIC JOURNAL OF MATHEMATICS
}

\author{
EDITORS
}

\author{
RICHARD ARENS (Managing Editor) \\ University of California \\ Los Angeles, California 90024
}

\author{
R. A. Beaumont \\ University of Washington \\ Seattle, Washington 98105
}

J. DugundjI

Department of Mathematics

University of Southern California

Los Angeles, California 90007

D. Gilbarg and J. Milgram

Stanford University

Stanford, California 94305

\section{ASSOCIATE EDITORS}
E. F. BECKENBACH
B. H. NEUMANN
F. WOLF
K. Yoshida

\section{SUPPORTING INSTITUTIONS}

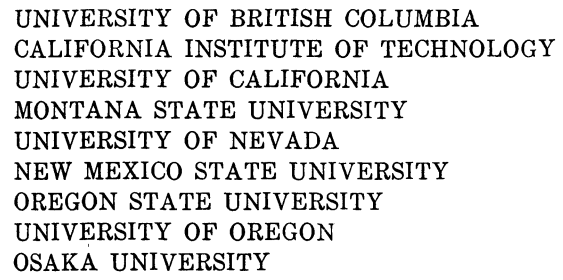

UNIVERSITY OF BRITISH COLUMBIA CALIFORNIA INSTITUTE OF TECHNOLOGY UNIVERSITY OF CALIFORNIA MONTANA STATE UNIVERSITY UNIVERSITY OF NEVADA NEW MEXICO STATE UNIVERSITY OREGON STATE UNIVERSITY UNIVERSITY OF OREGON OSAKA UNIVERSITY

\author{
UNIVERSITY OF SOUTHERN CALIFORNIA \\ STANFORD UNIVERSITY \\ UNIVERSITY OF HAWAII \\ UNIVERSITY OF TOKYO \\ UNIVERSITY OF UTAH \\ WASHINGTON STATE UNIVERSITY \\ UNIVERSITY OF WASHINGTON

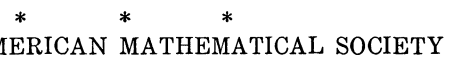

The Supporting Institutions listed above contribute to the cost of publication of this Journal, but they are not owners or publishers and have no responsibility for its content or policies.

Mathematical papers intended for publication in the Pacific Journal of Mathematics should be in typed form or offset-reproduced, (not dittoed), double spaced with large margins. Please do not use built up fractions in the text of your manuscript. You may however, use them in the displayed equations. Underline Greek letters in red, German in green, and script in blue. The first paragraph or two must be capable of being used separately as a synopsis of the entire paper. Items of the bibliography should not be cited there unless absolutely necessary, in which case they must be identified by author and Journal, rather than by item number. Manuscripts, in triplicate, may be sent to any one of the editors. Please classify according to the scheme of Math. Reviews, Index to Vol. 39. All other communications should be addressed to the managing editor, or Elaine Barth, University of California, Los Angeles, California, 90024.

The Pacific Journal of Mathematics expects the author's institution to pay page charges, and reserves the right to delay publication for nonpayment of charges in case of financial emergency.

100 reprints are provided free for each article, only if page charges have been substantially paid. Additional copies may be obtained at cost in multiples of 50 .

The Pacific Journal of Mathematics is issued monthly as of January 1966. Regular subscription rate: $\$ 72.00$ a year (6 Vols., 12 issues). Special rate: $\$ 36.00$ a year to individual members of supporting institutions.

Subscriptions, orders for back numbers, and changes of address should be sent to Pacific Journal of Mathematics, 103 Highland Boulevard, Berkeley, California, 94708.

PUBLISHED BY PACIFIC JOURNAL OF MATHEMATICS, A NON-PROFIT CORPORATION

Printed at Kokusai Bunken Insatsusha (International Academic Printing Co., Ltd.), 8-8, 3-chome, Takadanobaba, Shinjuku-ku, Tokyo 160, Japan.

Copyright (C) 1975 by Pacific Journal of Mathematics Manufactured and first issued in Japan 


\section{Pacific Journal of Mathematics}

\section{Vol. 63, No. 2 \\ April, 1976}

Joseph Anthony Ball and Arthur R. Lubin, On a class of contractive perturbations

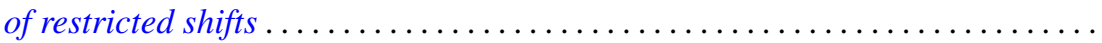

Joseph Becker and William C. Brown, On extending higher derivations generated

by cup products to the integral closure .......................

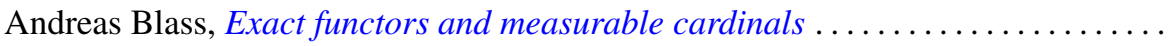

Joseph Eugene Collison, A variance property for arithmetic functions . . . . . . . . . .

Craig McCormack Cordes, Quadratic forms over nonformally real fields with a

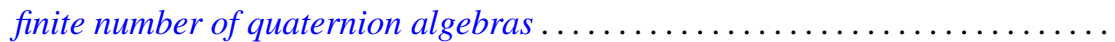

Freddy Delbaen, Weakly compact sets in $H^{1} \ldots \ldots \ldots \ldots \ldots \ldots \ldots \ldots \ldots$

G. D. Dikshit, Absolute Nörlund summability factors for Fourier series ..........

Edward Richard Fadell, Nielsen numbers as a homotopy type invariant. . ........

Josip Globevnik, Analytic extensions of vector-valued functions . . . . . . . . . . . .

Robert Gold, Genera in normal extensions . . . . . . . . . . . . . . . . . . . 389

Solomon Wolf Golomb, Formulas for the next prime

Robert L. Griess, Jr., The splitting of extensions of $S L(3,3)$ by the vector space

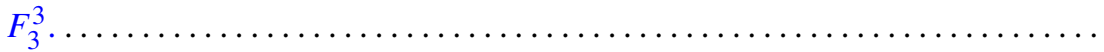

Thomas Alan Keagy, Matrix transformations and absolute summability .........

Kazuo Kishi, Analytic maps of the open unit disk onto a Gleason part.

Kwangil Koh, Jiang Luh and Mohan S. Putcha, On the associativity and commutativity of algebras over commutative rings . ..... . .

James C. Lillo, Asymptotic behavior of solutions of retarded differential difference equations.

John Alan MacBain, Local and global bifurcation from normal eigenvalues ..

Anna Maria Mantero, Sets of uniqueness and multiplicity for $L^{p}$

J. F. McClendon, Embedding metric families

L. Robbiano and Giuseppe Valla, Primary powers of a prime ideal .

Wolfgang Ruess, Generalized inductive limit topologies and barrelledness

properties.

Judith D. Sally, Bounds for numbers of generators of Cohen-Macaulay ideals

Helga Schirmer, Mappings of polyhedra with prescribed fixed points and fixed point indices.

Cho Wei Sit, Quotients of complete multipartite graphs

S. Sznajder and Zbigniew Zielezny, Solvability of convolution equations in $\mathscr{K}_{p}^{\prime}$,

$p>1$.

Mitchell Herbert Taibleson, The existence of natural field structures for finite

dimensional vector spaces over local fields

William Yslas Vélez, A characterization of completely regular fields

P. S. Venkatesan, On right unipotent semigroups ..............

Kenneth S. Williams, A rational octic reciprocity law ............

Robert Ross Wilson, Lattice orderings on the real field .......... 